\section{COMPROMISO TEMPORAL DISCRECIONAL: EFECTOS SOBRE LA ELECCIÓN Y EL ESTILO DE OCIO}

\author{
Robert A. Stebbins ${ }^{1}$ \\ University of Calgary
}

\section{DISCRETIONARY TIME COMMITMENT: EFFECTS ON LEISURE CHOICE AND LIFESTYLE}

\begin{abstract}
This paper introduces and elaborates the concept of discretionary time commitment, defined as un-coerced, allocation of a certain number of minutes, hours, days, or other measure of time that a person devotes, or would like to devote, to carrying out an activity. Discretionary time commitment finds expression in leisure and the agreeable sides of work (which, in effect, are experienced as leisure). The kinds of time commitments people make help shape their work and leisure lifestyles, thus constituting part of the patterning of those lifestyles. In leisure the nature of such commitments varies substantially across its three forms: serious, casual, and project-based leisure. In the field of leisure studies "time use" is generally treated of as an objective idea. Unlike the term "discretionary time commitment," it commonly connotes no subjective sense of personal agency or intentionality (to allocate time, set aside time, etc.).
\end{abstract}

KEY WORDS: Time commitment; leisure; serious leisure; casual leisure; project-based leisure.

El tiempo libre se ha considerado desde hace mucho en los Estudios de Ocio como un recurso clave que los participantes pueden manejar con miras a sus fines personales. También ha sido contemplado como una barrera fundamental para el ocio, que sin embargo puede ser negociada con otras personas. Y los estudios de usos del tiempo están ahí desde los comienzos de la investigación en ocio, considerados como un campo separado e interdisciplinar. Para arrojar nueva luz sobre este concepto capital, este artículo introduce otro denominado compromiso temporal discrecional.

\section{DEFINICIÓN DEL COMPROMISO TEMPORAL DISCRECIONAL}

El compromiso temporal discrecional es una asignación no forzada de cierto número de minutos, horas, días
RESUMEN: Este artículo introduce y elabora el concepto de compromiso temporal discrecional, que se define como la asignación no forzada de un cierto número de minutos, horas, días o cualquier otra unidad de tiempo que una persona consagra, o querría consagrar, a realizar una actividad. El compromiso temporal discrecional encuentra su expresión en el ocio y en las facetas agradables del trabajo (que, en la práctica, se experimentan como ocio). Los tipos de compromisos temporales que realizan las personas les ayudan a dar forma a sus estilos de vida laborales y de ocio, constituyendo así parte del modelado de estos estilos de vida. En el ocio, la naturaleza de estos compromisos varía sustancialmente en función de sus tres formas: ocio serio, casual o basado en proyectos. En el ámbito de los Estudios de Ocio se usa generalmente el "uso del tiempo" como una idea objetiva. Al contrario que la expresión "compromiso temporal discrecional", aquella no connota habitualmente ningún sentido subjetivo de iniciativa o intencionalidad personales (asignar tiempo, reservar tiempo, etc.).

PALABRAS CLAVE: Compromiso temporal; ocio; ocio serio; ocio casual; ocio basado en proyectos.

- cualquier otra unidad de tiempo que una persona dedica, o querría dedicar, a realizar una actividad. Tal compromiso es tanto proceso como producto. Esto es, las personas o bien establecen (proceso) sus propios compromisos temporales (productos), o bien aceptan voluntariamente tales compromisos (es decir, obligaciones agradables) establecidos para ellas por otros. Se sigue de aquí que las obligaciones desagradables, a las que las personas son forzadas invariablemente por otros o por las circunstancias, no constituyen compromisos temporales discrecionales, puesto que estos últimos, en cuanto procesos, descansan en la iniciativa personal. Dicho brevemente, el compromiso temporal discrecional encuentra su expresión en el ocio y en las facetas agradables del trabajo (esto es, "trabajo gustoso", que es experimentado en la práctica como ocio) (Stebbins, 2004a). 
Debe notarse, sin embargo, que podemos dedicar tiempo -y a veces lo dedicamos- a la realización de actividades desagradables, ya sea en el trabajo o fuera de él. Tales compromisos -llamémoslos compromisos temporales forzosos- son, desde luego, no discrecionales. En este sentido, quedan fuera del ámbito de esta reflexión y, con algunas interesantes excepciones, fuera del ámbito del ocio (es decir, ciertos costes del ocio -véase su más reciente discusión en Stebbins, 2007, cap. 1- pueden concebirse como compromisos temporales forzados).

En un sentido más general, comúnmente hablamos de compromisos temporales (discrecionales o forzados) pasados, presentes o futuros, en el trabajo, el ocio y en el ámbito de las obligaciones no laborales. Los tipos de compromisos temporales que realizan las personas les ayudan a dar forma a sus estilos de vida laborales y de ocio, constituyendo así parte del modelado de estos estilos de vida. En el ocio, la naturaleza de estos compromisos varía sustancialmente en función de sus tres formas: ocio serio, casual o basado en proyectos. Estas formas configuran la perspectiva del ocio serio, la base teórica principal para este artículo.

\section{LA PERSPECTIVA DEL OCIO SERIO}

La perspectiva del ocio serio (POS) es un marco teórico que sintetiza tres formas principales de ocio, mostrando, al mismo tiempo, sus rasgos distintivos, similitudes e interrelaciones (Stebbins, 2007). Las formas son el ocio serio, casual y basado en proyectos, brevemente definidas como sigue:

- Ocio serio: la práctica sistemática de una actividad amateur, de voluntariado o un hobby, suficientemente sustancial, interesante y plena como para que el participante encuentre ahí un itinerario (de ocio), centrado en adquirir y expresar la combinación de habilidades, conocimientos y experiencia específicos de la actividad.

- Ocio casual: actividad placentera inmediata e intrínsecamente gratificante, relativamente efímera, que requiere poco o ningún entrenamiento específico para su disfrute.

- Ocio basado en proyectos: desempeño creativo en el corto plazo, razonablemente complicado, realizado una única vez u ocasional pero infrecuentemente, que se lleva a cabo en el tiempo libre, o en el tiempo liberado de obligaciones desagradables.

La figura de la página siguiente es un mapa tipológico del mundo del ocio.

Por cuanto sabemos hasta el día de hoy, todo ocio (al menos en la sociedad occidental) puede clasificarse dentro de una de estas tres formas y sus varios tipos y subtipos. Más precisamente, la POS ofrece una clasificación y explicación de toda actividad y experiencia de ocio, en cuanto se enmarcan en los contextos socio-psicológicos, sociales, culturales e históricos en los que la actividad y la experiencia tienen lugar. La base empírica para esta perspectiva se expone en otro lugar (Stebbins, 2007; www.soci.ucalgary. ca/seriousleisure/Bibliography).

\section{OCIO SERIO}

Es el momento de completar la definición precedente. Así, los amateurs se distinguen de quienes practican un hobby por el hecho de que los primeros, puesto que pueden encontrarse en el arte, la ciencia, el deporte y el entretenimiento, tienen homólogos profesionales, mientras que los segundos no los tienen. Algunos practicantes de hobby, sin embargo, tienen homólogos comerciales. Sus cinco tipos se exponen en la figura 1. Los participantes en actividades incluyen gente que caza, practica canoa, recoge setas y observa pájaros. Los practicantes aficionados de deportes y juegos carecen de homólogos profesionales. Los hobbies vinculados a las artes liberales se basan en la auto-formación en un área de la vida o la literatura. Los voluntarios de ocio serio ofrecen ayuda, de un modo altruista y no forzado, ya sea formal o informalmente, sin ninguna retribución (o, como mucho, una retribución simbólica) y realizada en beneficio tanto de otras personas (más allá de la familia del voluntario) y del propio voluntario. Los seis tipos de voluntarios (fig. 1) son explicados en www.soci.ucalgary. ca/seriousleisure/digital library/Leisure Reflections16.

Todo ocio serio se define, además, por seis cualidades distintivas. Una es la eventual necesidad de perseverar, como a la hora de aprender cómo ser un guía de museo competente. Está claro que los sentimientos positivos so- 


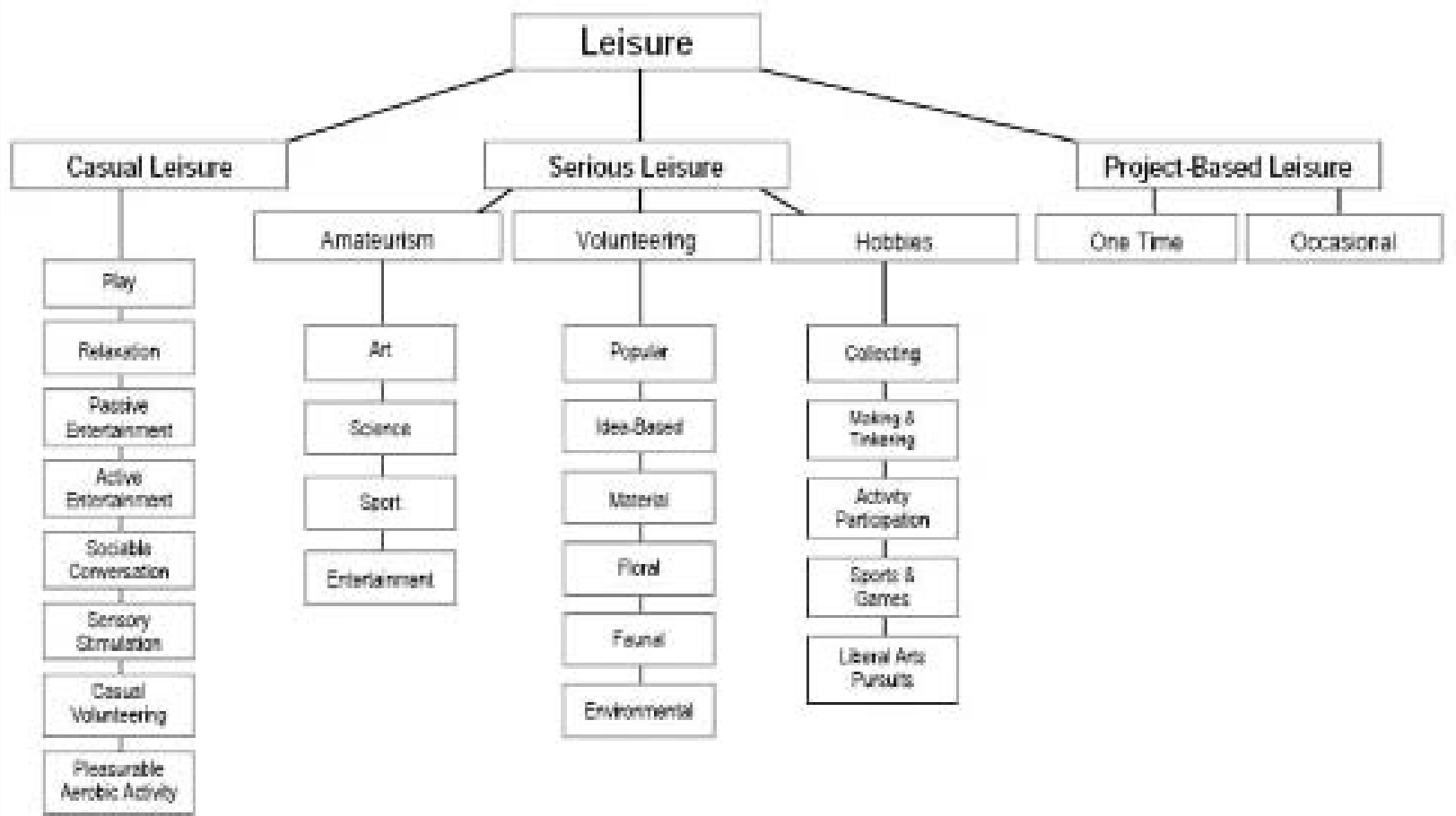

Disgran fartides by jeme Harts

bre la actividad provienen, hasta cierto punto, de aferrarse a ella contra viento y marea, de conquistar la adversidad. Una segunda cualidad consiste en encontrar una carrera en el rol de ocio serio, conformado por sus contingencias específicas, puntos de inflexión y escenarios de logro o implicación. Las carreras de ocio serio a menudo descansan sobre una tercera cualidad: esfuerzo personal significativo basado en conocimiento, entrenamiento, experiencia o capacidad específicamente adquiridos $y_{\text {, en ocasiones, }}$ de hecho, los cuatro a un tiempo. En cuarto lugar, hasta la fecha se han identificado varios beneficios duraderos, o resultados en sentido amplio; sobre todo a partir de la investigación sobre amateurs. Estos son auto-desarrollo, auto-enriquecimiento, auto-expresión, regeneración o renovación del self, sentimientos de logro, realce de la imagen de uno mismo, interacción social y pertenencia, y productos físicos perdurables de la actividad (por ejemplo, una pintura, un artículo científico, un mueble). Otro beneficio es el de la auto-gratificación, o la combinación de disfrute superficial y profunda realización. El más potente de todos estos beneficios es la autorrealización -realizar, o el acto de haber realizado, al máximo los propios talentos y carácter, el propio potencial-.

Una quinta cualidad del ocio serio es el ethos único que se genera alrededor de cada uno de sus casos. Un componente central de este ethos es un mundo social específico en el que los participantes persiguen sus intereses de tiempo libre. Unruh $(1980,277)$ desarrolló la siguiente definición:

Un mundo social debe verse como una unidad de organización social que es difusa y amorfa en su carácter. Por lo general más grandes que grupos $u$ organizaciones, los mundos sociales no quedan definidos necesariamente por límites formales, listas de miembros o territorio espacial (...). Un mundo social debe verse como una constelación de agentes, organizaciones, eventos y prácticas internamente reconocible que se ha fusionado en una esfera percibida de

ARBOR Vol. 188754 marzo-abril [2012] 293-300 ISSN: 0210-1963 
intereses e implicación para los participantes. Caracteristicamente, un mundo social carece de una potente estructura de autoridad centralizada y está delimitado por (...) la comunicación efectiva y no por el territorio o la pertenencia formal al grupo.

La sexta cualidad descansa en las cinco precedentes: los participantes de ocio serio tienden a identificarse fuertemente con sus actividades elegidas.

\section{Ocio casual}

El ocio casual, del cual hay ocho tipos, carece de estas seis cualidades (ver figura 1). La última y más reciente adición a esta tipología -la actividad aeróbica placentera- refiere a actividades físicas que requieren esfuerzo suficiente como para causar un marcado incremento en la frecuencia cardíaca y la respiración (Stebbins, 2004b). Nos referimos aquí a "actividad aeróbica" en su sentido amplio, a toda actividad que demanda tal esfuerzo. Así, el concepto incluye las rutinas practicadas colectivamente en las clases de aeróbic y todas aquellas practicadas individualmente por medio de programas de aeróbic televisados o en vídeo. Como el resto de tipos de ocio casual, este tipo requiere sólo mínimas capacidades, conocimientos o experiencia.

\section{OcIO BASAdO EN PROYECTOS}

Aunque la exploración sistemática puede revelar otros, han sido dos los tipos de ocio basados en proyectos identificados hasta el momento.

\section{Proyectos de carácter puntual}

En todos estos proyectos las personas usan los talentos y el conocimiento de que disponen, aunque para algunos proyectos pueden necesitar buscar formación específica. Esto podría incluir la lectura de un libro o la asistencia a un curso. Y ciertos proyectos pueden requerir algún acondicionamiento preliminar. El objetivo consiste siempre en realizar exitosamente el proyecto único y nada más, y en ocasiones se hace necesaria para esto una cierta preparación básica. Es posible que una encuesta mostrase que la mayor parte del ocio basado en proyectos tiene naturaleza de hobby, mientras que el siguiente tipo más común sería una clase particular de voluntariado. En primer lugar, se han identificado los siguientes proyectos de tipo hobby:

\section{- Hacer manualidades y reparaciones:}

$\square$ Kits para entrelazar, encajar piezas y hacer nudos.

$\square$ Otros proyectos de kits de ensamblaje (por ejemplo, radio estéreo, proyectos de manualidades).

口 Proyectos del tipo de "hágalo Vd. mismo", realizados con miras a la satisfacción, algunos de los cuales pueden requerir la obtención de ciertas habilidades o conocimientos preliminares (por ejemplo, construir un muro de piedra, acabar un cuarto de juegos, plantar un jardín especial). Esto puede convertirse en una serie irregular de tales proyectos, a lo largo de muchos años. Estos pueden transformar al participante en un practicante de hobbies.

- Artes liberales:

$\square$ Genealogía (no como un hobby continuo).

$\square$ Turismo: un viaje especial, que no sea parte de un extenso programa turístico personal para visitar diversas partes de una región, un continente o el mundo entero.

- Participación en actividades: un largo viaje como "mochilero", viaje en canoa; un ascenso único a una montaña (por ejemplo, Fuji, Rainier, Kilimanjaro).

También son frecuentes los proyectos de voluntariado de carácter puntual, aunque posiblemente algo menos que los proyectos de quienes cultivan hobbies. Y menos comunes incluso que los proyectos de tipo amateur, que parecen ser principalmente teatrales.

\section{- Voluntariado}

$\square$ En una convención o congreso.

$\square$ En una competición deportiva.

$\square$ En un festival artístico o una exposición temporal en un museo.

$\square$ Ayudando a restablecer las condiciones de vida, humanas o animales, tras un desastre natural o provocado por el hombre, como, por ejemplo, un huracán, 
un terremoto, un vertido de petróleo o un accidente industrial.

- Proyectos artísticos (esta nueva categoría reemplaza a "Teatro de entretenimiento") (vid. Stebbins, 2011):

$\square$ Teatro de entretenimiento: realizar una puesta en escena o una edición de un desfile o cabalgata comunitaria; preparar una película, video o álbum fotográfico caseros.

$\square$ Intervenciones en público: preparar una charla para una reunión, un discurso tras una cena, una ponencia o un asunto a ser debatido dentro de un grupo de encuentro.

$\square$ Memorias: producciones audiovisuales o escritas, de carácter terapéutico, realizadas por personas mayores; historias de vida y autobiografías (todas las edades); relato de sucesos personales (todas las edades) (Stebbins, 2011).

\section{Proyectos ocasionales}

La observación preliminar sugiere que, comparados con aquellos de tipo puntual, es más probable que los proyectos ocasionales se originen en o estén motivados por una obligación agradable. Los ejemplos de proyectos ocasionales incluyen la suma de actividades creativas culinarias, decorativas y otras, emprendidas, por ejemplo, en casa o en el trabajo, con ocasión de una festividad religiosa o el cumpleaños de alguien. Igualmente, las fiestas nacionales o celebraciones semejantes inspiran a veces a las personas a la hora de organizar proyectos ocasionales, que se distinguen por lo surtido de sus elementos inventivos.

\section{Compromisos temporales VARIABLES}

En términos generales, el ocio serio exige a sus participantes dedicarle más tiempo que en el caso de los participantes en cualquiera de las otras dos formas, siquiera sea porque, de los tres, el ocio serio parece ser practicado con mayor frecuencia a lo largo del lapso de tiempo más extenso. Además de esto, determinadas cualidades del ocio serio, incluyendo especialmente la perseverancia, el com- promiso, el esfuerzo y el itinerario, tienden a hacer que los amateurs, los practicantes de un hobby, así como los voluntarios, tomen conciencia acerca de cómo ellos mismos emplean su tiempo libre, la cantidad de él que usan para su ocio serio y los modos como lo consiguen.

Hay muchos ejemplos. Las actividades de amateurs y practicantes de un hobby que están basadas en el desarrollo y perfeccionamiento de habilidades físicas (por ejemplo, aprender a hacer malabares, patinaje artístico, confección de colchas, o a tocar el piano) requieren del aspirante a entretener, patinar, tejer, etc., comprometer un buen número de horas de forma regular, en ocasiones a lo largo de muchos años, para poder adquirir y pulir las habilidades necesarias. $Y$ una vez las habilidades, $y$ el acondicionamiento físico relacionado con las mismas, han sido adquiridos, deben ser mantenidos a través del uso continuo. Además, algunos entusiastas del ocio serio asumen (agradables) obligaciones (Stebbins, 2000b) que demandan su presencia en ciertos lugares a ciertas horas (por ejemplo, ensayos, partidos, encuentros, eventos). Pero lo que es más importante, la actividad central, que es la esencia del ocio serio de una persona, resulta tan atractiva, que este individuo desea reservarse tiempo suficiente para entregarse a ella.

En otras palabras, el ocio serio a veces amenaza con ser incontrolable. Engendra en algunos practicantes un deseo de practicar la actividad más allá del tiempo o el dinero disponibles para ella (cuando no ambos). Así, aunque hobbies tales como coleccionar sellos o fabricar muebles por lo común tienen pocas citas u oportunidades en que congregar a sus aficionados, éstas tienen sin embargo un enorme atractivo, y animan a estos coleccionistas y bricoleurs a dedicar, cuando es posible, tiempo para ese ocio.

El ocio basado en proyectos puede venir acompañado de demandas similares. Es un desempeño creativo en el corto plazo, razonablemente complicado, realizado una única vez u ocasional pero infrecuentemente, que se lleva a cabo en el tiempo libre, o el tiempo liberado de obligaciones desagradables (Stebbins, 2000b). En él, los participantes pueden encontrar reuniones o responsabilidades programadas (cuando no ambas cosas), y a pesar de su corto alcance, su condición incontrolable puede llegar a ser también una preocupación. Pero el ocio basado en proyectos no incluye, por definición, el desarrollo, el perfeccionamiento y el 
mantenimiento de las habilidades físicas, lo que constituye una de las diferencias clave del uso del tiempo discrecional, distinguiéndolo del ocio serio.

Además, el ocio basado en proyectos comporta una sensación única de asignación temporal: el empleo del tiempo es más o menos intenso, pero está limitado a un período conocido y marcado en el calendario (por ejemplo, cuando se termina una competición atlética, cuando el muro de piedra está construido, cuando la fiesta sorpresa de cumpleaños ha tenido lugar). De hecho, para ciertas personas, uno de los atractivos de los proyectos es que no está previsto un compromiso temporal de largo aliento.

Finalmente, el ocio casual, a su propio modo, puede generar compromisos temporales, como el deseo de reservar una hora cada semana para ver un programa de televisión o participar, tan frecuentemente como sea posible, en un café matutino con el vecindario. Además, cierto ocio casual, el más popular puede ser ver la televisión, es atractivo, en parte, porque a menudo está disponible en cualquier momento -llámeselo compromiso espontáneo de tiempo discrecional-; pudiendo rellenar los saltos entre compromisos temporales, discrecionales y forzosos, y en el proceso evitar el aburrimiento. Por añadidura, el voluntariado ocasional presenta comúnmente requerimientos temporales, como al sumarse a una cuadrilla de limpieza medioambiental durante el fin de semana, al servir a los pobres comidas gratis el día de Acción de Gracias, al recolectar dinero para obras de caridad yendo de puerta en puerta o pidiendo en la calle.

Por otra parte, al diseñar sus estilos de ocio, las personas mezclan y coordinan su participación en, y su dedicación de tiempo libre $a$, una o más de estas tres formas. Bajo esta mirada, algunas personas intentan organizar su tiempo libre de tal forma que se acercan, tal y como lo definen, a un "óptimo estilo de ocio" (Stebbins, 2000a). El término se refiere a la práctica profundamente gratificante e interesante, durante el tiempo libre, de una o más formas sustantivas y absorbentes de ocio serio, complementadas con cantidades prudentes de ocio casual u ocio basado en proyectos, o de ambos. La gente halla estilos de ocio óptimos al tomar parte de actividades de ocio que, individualmente y en combinación, les ayudan a desarrollar su potencial humano, conduciéndolos de ese modo a la autorrealización y a la mejora del bienestar y la calidad de vida.
La asignación discrecional de tiempo en el ocio es, por tanto, altamente complicada. Así, al abordar el tema, debemos estar seguros de especificar la forma $y$, dentro de la forma, el tipo de ocio en cuestión. La asignación de tiempo difiere sustancialmente entre el boxeo amateur y el aficionado al canto a capella (Barbershop singing), entre éste y el voluntario en la lucha contra el fuego, o entre dedicarse a realizar árboles genealógicos o a la contemplación casual de personas desde un café a pie de calle. La complejidad de la asignación de tiempo de ocio puede también quedar afectada por otras personas significativas. No es infrecuente que tal asignación se negocie con cónyuges, compañeros, amigos y parientes. El participante en ocio puede querer dedicar a una actividad más tiempo de lo que esas personas están dispuestas a aceptar, dado que estas últimas desean que aquel gaste tiempo (y quizá dinero) con ellos. Nuevamente, se introduce el asunto de la "incontrolabilidad".

\section{El estudio del TIEMPO EN OCIO}

En los Estudios de Ocio, el término "uso del tiempo", de forma diversa a nuestro término "compromiso de tiempo discrecional", no connota habitualmente ningún sentido subjetivo de intencionalidad o iniciativa personales (asignar tiempo, reservar tiempo, etc.). El uso del tiempo, tal y como se concibe convencionalmente, es una idea objetiva. Normalmente se asumen dos niveles de análisis diferentes, ambos generales. 1) Uso del tiempo participativo, como en la participación en actividades, se refiere a las cantidades promediadas de tiempo que las diferentes categorias de personas dedican a actividades concretas (a menudo expresadas como patrones de uso del tiempo). 2) Uso general del tiempo, como en las asignaciones globales de tiempo a lo largo de un día o una semana típicos, se refiere a cómo las diferentes categorías de personas dedican típicamente su tiempo tanto al ocio como al trabajo, al igual que a otras obligaciones no laborales.

El compromiso temporal discrecional también es particular; pero, de todos modos, gira alrededor de cómo los individuos tienden a dedicar su tiempo como parte de su estilo de vida $y_{1}$ en este artículo, de cómo se involucran en el ocio en general, y en ciertas actividades de ocio más en particular. El compromiso de tiempo discrecional es subjetivo. Tiene directamente que ver con la motivación y la inclinación a 
participar. Así, se trata de un concepto individualista, que cuando se generaliza, se expresa como el estilo de vida de un determinado tipo de participantes del ocio (por ejemplo, el estilo del voluntario en tareas de búsqueda y rescate de trabajadores, de "kayakistas" aficionados, de "teleadictos", de astrónomos amateur). Por contraste, el uso del tiempo de ambos tipos es algo cultural $y$, por tanto, inevitablemente general; lo individual es de escasa relevancia, excepto como fuente de recopilación de datos en orden a fijar patrones y tendencias de alcance más amplio.

Más aún, dentro de sus estilos de vida la gente mezcla y coordina su participación y su asignación de tiempo libre al involucrarse en una o más de las tres formas de ocio. Y algunas personas van todavía más lejos, al intentar organizar su tiempo libre, y asi beneficiarse de este hecho, por medio de lo que anteriormente hemos descrito como un estilo de ocio óptimo. En breve, la mayor parte de las personas administran activamente esta parte de sus vidas, intentando crearse en el curso de ella una existencia digna.

Debido a que el ocio casual resulta muy popular y tiende así a formar parte de la cultura general, por inferencia podemos aprender de los estudios de uso del tiempo algo acerca del compromiso temporal discrecional a dicho nivel. Esto no es válido, en cualquier caso, para el ocio serio o el ocio basado en proyectos. Éstos son mucho menos populares, y a la vez parecen presentar patrones de compromiso temporal discrecional mucho más complicados. Los estudios generales del uso del tiempo, al menos tal y como han sido realizados en el pasado, nos dicen muy poco acerca de cómo se ha asignado el tiempo discrecional en estas dos formas.

\section{Conclusión: GeneralizAR UN ESTILO DE VIDA}

De acuerdo a Veal (2003), la investigación sobre estilos de vida declinó durante los 80 y los primeros 90, pero volvió a cobrar fuerza en la segunda mitad de los 90, alentada por el interés en los estilos de vida consumistas (véase Veal, 2000, con una útil bibliografía online anotada de obras que tratan los estilos del ocio). Pero, tal y como Kelly (1999) ha observado, las clasificaciones de estilos de vida que se fijaban en el consumo suponen una mirada muy estrecha; por esta razón, fallan a la hora de arrojar luz teórica suficiente sobre la naturaleza general del estilo de ocio. Más precisamente, el problema en el pasado ha sido cómo escapar de la tendencia idiográfica en la investigación sobre estilos de vida, es decir, la tendencia a informar en detalle sobre un estilo de vida en concreto, digamos el de los tenistas amateurs, el de los voluntarios en la lucha contra el fuego, o el de los seguidores de telenovelas, mientras que se hacía muy poco esfuerzo por generalizar dicho estilo de vida a la categoría más amplia dentro de la cual cada uno de estos entusiastas figuraría como atleta, voluntario o telespectador. Al mostrar cómo el proceso de comprometer tiempo discrecional varía a lo largo de cada una de las tres formas de ocio, he intentando superar esta debilidad, con la intención de aportar a la teoría y la investigación una manera de observar el estilo de vida que favorezca la generalización.

Quizás, con esta aproximación, pueda revertirse el declive percibido por Veal, no sólo por lo que atañe a la investigación sobre estilos de consumo, sino también sobre estilos de ocio en general.

\section{NOTA}

1 Nota de los traductores: Este artículo se presenta en traducción al castellano con el conocimiento y la aprobación del autor, suponiendo un tratamiento inédito, actualizado y ampliado, de un texto anterior aparecido en Leisure Studies Association Newsletter: "Leisure Reflection No 12". Traductores: Jaime Cuenca y Fernando Bayón.

\section{BIBLIOGRAFÍA}

Kelly, John R. (1999): "Leisure behaviors and styles: Social, economic, and cultural factors", en Leisure Studies: Prospects for the Twenty-First Century (Edgar L. Jackson and Thomas L. Burton, ed.), State College, PA: Venture, pp. 135-150.

Stebbins, Robert A. (2000a): "Optimal leisure lifestyle: Combining serious and casual leisure for personal well-being", en Leisure and Human Development:
Aceptado: 14 de julio de 2011

doi: 10.3989/arbor. $2012.754 \mathrm{n} 2003$ 
Proposals for the 6th World Leisure Congress (editado por Manuel C. Cabeza), Bilbao: Universidad de Deusto, pp. 101-107.

Stebbins, Robert A. (2000b): "Obligation as an aspect of leisure experience", Journal of Leisure Research, 32, 152 155.

Stebbins, Robert A. (2004a): Between Work and Leisure: The Common Ground of Two Separate Worlds, New Brunswick, NJ: Transaction.

Stebbins, Robert A. (2004b): "Pleasurable Aerobic Activity: A Type of Casual Leisure with Salubrious Implications", World Leisure Journal, 46(4), 55-58 (también disponible en www.soci.ucalgary.cal seriousleisure-Digital Library, Other Works).

Stebbins, Robert A. (2005): Project-based leisure: Theoretical neglect of a common use of free time. Leisure Studies, 24, 1-11.

Stebbins, Robert A. (2007): Serious Leisure: A Perspective for Our Tim, New Brunswick, NJ: Transaction.

Stebbins, Robert A. (2011): "Personal memoirs, project-based leisure and therapeutic recreation for seniors", en Leisure Studies Association Newsletter, 88 (March), pp. 29-31 (también en www.soci.ucalgary.ca/seriousleisure-
Digital Library, "Leisure Reflections No. 26").

Unruh, David R. (1980): "The nature of social worlds", Pacific Sociological Review, 23, 271-296.

Veal, A. J. (2000): Lifestyle and leisure: A review and annotated bibliography, Sydney, Australia: School of Leisure and Tourism Studies, University of Technology, www.business.uts.edu.au/ leisure/research/bibs.html.

Veal, A. J. (2003): "Lifestyle", en Encyclopedia of Leisure and Outdoor Recreation (editado por John M. Jenkins and John J. Pigram), London: Routledge, p. 288. 